Published in final edited form as:

J Opt Soc Am A Opt Image Sci Vis. 2014 April 1; 31(4): A314-A321.

\title{
Dynamics of Color Contrast Adaptation
}

\author{
Katherine Tregillus and Michael A. Webster \\ Department of Psychology, University of Nevada, Reno NV 89557 USA
}

\begin{abstract}
Many forms of color adaptation have been found to reflect both short- and long-term adjustments. We explored the buildup and decay of adaptation to chromatic contrast (temporal modulations of color) for which the dynamics are unknown. A matching task was used to track the perceived contrast of chromatic pulses of varying physical contrast during and after adapting for one hour to a high contrast modulation repeated over 5 successive days. The adaptation was characterized by rapid response changes that remained stable in both time course and form across sessions. There was no consistent evidence for long-term plasticity over the timescales we tested.
\end{abstract}

\section{Introduction}

The processes of adaptation strongly influence most aspects of visual coding and have been extensively studied, yet the basic dynamics of adaptation remain poorly characterized [1]. Most work has focused on sensitivity changes operating over short time scales, from milliseconds to minutes [2, 3]. However, there is growing evidence that response changes also occur over much longer periods. In color, long-term aftereffects are well known from the McCollough effect (ME), the orientation-contingent color afterimages induced in achromatic gratings after adapting to horizontal and vertical gratings paired with different colors $[4,5]$. These aftereffects include not only a transient color change but also color biases that may persist permanently until the observer is actively "deadapted" by exposing them to a different color and orientation combination [6]. Long term aftereffects of chromatic adaptation have also been found following prolonged adaptation to a color bias created by changing the average lighting in an observer's environment, by wearing tinted contact lenses, or by viewing a constant color presented in a pattern such as randomly changing lines [7-9]. Each of these procedures can lead to aftereffects in unique yellow that can last for days or weeks. Similar very slow appearance changes are also found in cataract patients, who adapt to the yellowing of their lens. After the lens is removed, achromatic settings can again show both rapid and very prolonged adjustments that can take months to return to their presurgery settings $[10,11]$. Thus long term aftereffects appear to be a common characteristic of color adaptation.

However, whether the dynamics of color aftereffects reflect common underlying mechanisms is not well understood. Color adaptation includes multiple distinct sites and forms of sensitivity adjustments. These include cone-specific gain changes in the receptors, "second-site" adjustments within post-receptoral mechanisms, and adaptation to chromatic contrast - to the spatial or temporal variations in color - within cortical mechanisms [12]. Though largely monocular, the ME is itself thought to reflect a cortical response change since this is the first stage at which visual neurons become strongly selective for orientation [5]. Other long term color changes have also been attributed to response changes arising in the cortex $[7,10]$. Yet at least some forms of long-term sensitivity regulation appear to operate as early as the photoreceptors [13]. In this study we explored the dynamics of chromatic contrast adaptation. Viewing a field that alternates in time between two chromaticities, or to a pattern such as grating that alternates between two colors over space, 
produces large and selective losses in sensitivity to the color variations along the adapting axis [14-18]. For example, after adapting to a reddish-greenish modulation, reds and greens appear less saturated. These adjustments may play an important role in matching neural responses to the gamut of color (as opposed to simply the average color) in an observer's environment, and thus are likely to play a critical role in visual coding [12] The time course of this adaptation is thus important to characterize, but has not been explored previously. As noted, contrast adaptation is distinct from simple chromatic adaptation to the average color, and primarily reflects response changes at a cortical level [19]. Yet how the effect is related to other cortical color aftereffects like the ME remains unclear. For example, color contrast adaptation is also selective for orientation [17, 18], and can result in hue biases reminiscent of the color shifts conventionally measured in the ME [14, 20]. However, it remains possible that it only plays a role in the transient component of the color biases in the ME [6]. Characterizing the dynamics of chromatic contrast adaptation can thus help clarify how they might be related to phenomena such as the ME and its persistence. A first goal of our study was to test whether temporal contrast adaptation can support a long-term aftereffect.

While few studies have explored these questions for chromatic contrast, there has been important work on the dynamics of adaptation to luminance contrast, with most of this focused on spatial contrast [21-23]. Recent studies have documented long-term adjustments to patterns of luminance contrast. For example, Zhang et al. [24] found that four or more hours of exposure to scenes in which contrasts were filtered to remove energy at certain orientations led to persistent biases in orientation coding. Moreover, these aftereffects showed spontaneous recovery from transient aftereffects produced by brief adaptation to an opposing pattern, suggesting that there are distinct mechanisms of short and long term adaptation [25]. Similar dissociations have also been found for other pattern-selective aftereffects including changes in the perceived direction of motion or the perceived distortions in images of faces [26]. This again suggests the possibility that adaptation in general operates over separate and multiple timescales. Our study was partly motivated to explore whether there were analogous long-term aftereffects in adaptation to chromatic contrast.

A further important and unanswered question is whether the timescale of the adaptation can influence how visual sensitivity changes. Kwon et al. [27] recently explored this by measuring spatial contrast coding in observers who wore contrast-reducing goggles. After 4 hours of adaptation their contrast sensitivity improved. Moreover, they found that the form of the responses changes shifted from contrast gain, which characterizes contrast adaptation at short timescales, to response gain, and suggested that the short and long response changes were adjusting to different aspects of the stimulus (specifically to the mean contrast at brief intervals while to the maximum contrast at longer intervals). Webster, McDermott and Juricevic [28-30] have similarly argued from analyses of images simulating "theoretically complete" states of adaptation that the visual system should adjust with multiplicative (response gain) changes at long timescales rather than the subtractive sensitivity changes typically seen at short durations [31], in order to match contrast coding for the gamut of contrasts in different environments. Thus, not only is there evidence for multiple rates of adaptation but also for duration-dependent changes in the type of adaptation. As a second question, we asked whether the form of chromatic contrast adaptation changed as observers adapted for longer periods.

With repeated adaptation, there is also the possibility that the dynamics themselves change. For example, Yehezkel et al. [32] examined how observers adapt to astigmatic blur. When observers were first exposed to the blur, they required substantial time to adjust to the distortion, and showed strong aftereffects when the blur was removed. However, with 4 hours of adaptation, on subsequent days the adjustments were instead almost immediate, and 
aftereffects from the blur were diminished. This suggests a form of learning or contingent adaptation in which observers might store different adaptation states for the different viewing conditions, so that they could rapidly switch to the appropriate calibrationfor the current context. As a final aim, we also asked whether observers could "learn" to adapt to chromatic contrast, by testing whether the dynamics of adaptation can change when observers are repeatedly exposed to the same adapting stimulus.

\section{Methods}

\section{Participants}

Observers included the authors and 5 additional individuals who were unaware of the specific aims of the study. These naïve observers and author KT had not previously participated in color contrast adaptation experiments and had not been previously exposed to the specific adapting modulation used in the study. Such observers were chosen to explore whether the dynamics might change with repeated adapting sessions. MW has instead had very extensive experience in adaptation studies with these stimuli, spanning roughly 25 years [14]. All observers had normal color vision as assessed by the Cambridge Colour Test, and normal or corrected-to-normal visual acuity. Participation was with informed consent and followed protocols approved by the university's institutional review board.

\section{Stimuli}

The stimuli were presented on a SONY 20SE monitor controlled by a Cambridge Research Systems VSG board, which allows colors to be displayed with high resolution. The display was calibrated with a Photo Research PR650 spectroradiometer, with gun outputs linearized through look-up tables. The spatial display consisted of two uniform 4-deg squares, centered $2.5 \mathrm{deg}$ above or below a central fixation point. The adapt and test stimuli were shown alternated within the upper field, while the matching stimulus was shown in the lower field and displayed concurrently with the test stimulus. Both fields were shown on a 15 by $20 \mathrm{deg}$ uniform gray background corresponding to the full screen of the monitor, and were delimited from the background by static narrow black borders. The gray background had a luminance $\left(30 \mathrm{~cd} / \mathrm{m}^{2}\right)$ and chromaticity equivalent to the mean level of the adapting stimulus. Observers viewed the display binocularly from a distance of $114 \mathrm{~cm}$.

The experiments probed changes in perceived contrast along the $\mathrm{L}$ vs. $\mathrm{M}$ axis of coneopponent space, which corresponds to opposing modulations within the $\mathrm{L}$ and $\mathrm{M}$ cones at constant luminance and at a constant level of $\mathrm{S}$ cone excitation. Contrast was defined as the distance along this chromatic axis relative to the chromaticity of the (zero contrast) gray background, based on a scaled-version of the cone-opponent space. The reported units for our stimuli are related to the the $1, \mathrm{~s}$ coordinates of the MacLeod-Boynton chromaticity diagram by:

$$
\begin{aligned}
\text { LvsM contrast } & =(1-0.6568) * 2754 \\
\text { SvsLM contrast } & =(\mathrm{s}-0.01825) * 4099
\end{aligned}
$$

The scaling constants were based on a previous study ([33]) in which contrast was scaled as multiples of the threshold for detecting a color change from the gray background, though we did not measure contrast thresholds as part of the current study. As noted, in the current study we also varied only the LvsM contrast, and thus the SvsLM contrast remained zero (at the level of the gray background, which had MacLeod-Boynton coordinates of .6568, . 01825, equivalent to the chromaticity of illuminant C). 
Adapting stimuli corresponded to $1 \mathrm{~Hz}$ sinusoidal modulations over a range of \pm 80 contrast units. Test stimuli were presented as brief pulses of color along the $+\mathrm{L}$ axis with a full contrast of 20,40 , or 80 units, and thus varied from $25 \%$ to $100 \%$ of the adapting contrast level. The pulses were tapered with a $100 \mathrm{msec}$ sinusoidal onset and offset to avoid temporal transients.

\section{Procedure}

To track the changes in perceived contrast as a function of the adapting time, we used a procedure similar to one described by Vul et al. [6]in their study of the McCollough effect. Throughout a session the test stimuli were shown every $5 \mathrm{sec}$, with the three contrast levels alternated. Thus a single contrast (e.g. 20 units) was presented every $15 \mathrm{sec}$. Observers were first shown the test stimuli under neutral adaptation to a gray field (pre-adapt phase) for a period of $120 \mathrm{sec}$. During this time they adjusted the contrast of the color pulse shown simultaneously in the matching field so that it appeared to have the same contrast as the test pulse. During the adapt phase, the neutral field was instead replaced with the adapting flicker, with a brief $(0.5 \mathrm{sec})$ gray interval between the adapt phase and test. Observers continued to track the perceived contrast of the test stimuli throughout the adaptation period for one hour. Finally, during the post-adapt period the adapt stimulus was returned to a uniform gray field and the recovery of perceived contrast was tracked for a further $600 \mathrm{sec}$. (The entire session required maintaining reasonable fixation to the center of the display throughout, a task which was arduous but which observers were instructed to adhere to.) Adapt sessions were repeated for 5 successive days. For most observers, these were run on consecutive days, though for some observers up to 8 days were necessary to complete the full set. Our results do not reveal differences related to the total experimental duration.

\section{Results}

Figure 1 (color online) show representative examples of the full set of measurements for 4 of the observers. In each panel, the traces track the matches for the 3 different contrast levels over the 5 successive sessions, and include settings prior, during, and after the daily adapting exposure. For each observer, the perceived contrast of the test stimuli is reduced rapidly during the adaptation and recovers rapidly when the adaptation ended. The pattern is roughly similar across the different contrast levels, and remains roughly similar across the 5 sessions, so that the results across the 5 days are largely superimposed.

Settings at each day and contrast level were fit with exponentials to estimate the changes in perceived contrast and the time constants of the adaptation. Separate fits were made to each experimental phase (i.e. pre-, during, and post- adapt), and were based on the following equation:

$$
\mathrm{C}_{\mathrm{sec}}=\mathrm{C}_{\max }+\left(\mathrm{C}_{\min }-\mathrm{C}_{\max }\right)\left(1-\mathrm{e}^{(-(\mathrm{sec} / \tau))}\right)
$$

where $\mathrm{C}_{\mathrm{sec}}$ is the estimated LvsM contrast (as defined in equation 1) at the time from the start of the phase (sec), $\mathrm{C}_{\min }$ and $\mathrm{C}_{\max }$ are the minimum and maximum LvsM contrast, and $\tau$ is the time constant of the decay or recovery. Values of $\mathrm{C}_{\min }, \mathrm{C}_{\max }$ and $\tau$ were based on a least-squares fit of the predicted to measured contrast settings. Figure 2 shows examples of these fits for KT and MW's settings. For most observers like KT, a single exponential was sufficient to characterize the contrast changes for a given adapting condition. MW was instead unique among the observers in showing a steady buildup of the adaptation throughout the adapting interval, in addition to the rapid initial change characterizing all observers' settings. As a result his settings are not well described by a single exponential function that asymptotes rapidly. This prolonged buildup was verified by fitting linear 
regression lines to the settings during the final 50 minutes of the session (with the first 10 minutes excluded to exclude the initial response changes). The slopes of these functions (perceived contrast vs. secs adapt) across the 5 days averaged $-.00096(\mathrm{sd}=.00015),-.0025$ $(\mathrm{sd}=.00064)$, and $-.00046(\mathrm{sd}=.00026)$ for the test contrasts of 20,40 , or 80 respectively. The mean slopes at each test contrast were all significantly different from zero $(\mathrm{t}(4)>=8.81$, $\mathrm{p}<=.0009)$, but notably did not differ from each other when expressed as a percent change in contrast $(F(2,12)=2.76, p=0.1)$. None of the other observers exhibited slopes that significantly differed from zero.

For all observers, we used estimates from the exponential fits to ask whether there was evidence for any residual long-term change in perceived contrast, either by the end of the post-adapt period within the session or during the pre-adapt phase of the subsequent session. This was assessed by comparing the contrast matches at the beginning or end of each session. For each the perceived contrast was estimated from the end of the pre-adapt or postadapt phase, and was based on the fitted exponential at that point. Figure 3 shows the mean values for these matches averaged across all observers. In each panel the 3 curves are again for the 3 different contrast levels. For each, the perceived contrast at both the start and end of the session did not differ from a physical match for any of the test contrasts (as assessed by one-sample $\mathrm{t}$-tests $(\mathrm{t}(34)<1.65, \mathrm{p}=.12)$ ), and there was no evidence for a change in the percept as the adaptation days progressed (pre-adapt: $\mathrm{F}(4,48)=1.62, \mathrm{p}=0.20$; post-adapt: $\mathrm{F}(4,48)=0.412, \mathrm{p}=0.80)$. Thus there were not measureable aftereffects by the end of the 600 sec post-adapt interval within each day, and moreover there was not a significant carry-over of the aftereffects across successive days.

We next evaluated whether the rate of adaptation and recovery changed across trials. To assess this, we compared the time constants across successive days. For this analysis MW's settings were excluded because the single exponential fits failed to characterize the adaptation time course. For the post-adapt we excluded one additional subject due to variability in their settings. The mean values across observers are plotted in Figure 4, again for the different contrast levels and as a function of the session number. Time constants across the three contrast levels averaged $136 \mathrm{sec}$ during adaptation and $99 \mathrm{sec}$ during the post-adapt recovery. For the post-adapt recovery they did not differ significantly for the different test contrast levels $(\mathrm{F}(2,32)=1.22, \mathrm{p}=0.34)$, and did not significantly change with the adapting session $(\mathrm{F}(4,32)=0.48, \mathrm{p}=0.75)$. During adaptation there was no effect of contrast $(\mathrm{F}(2,40)=1.43, \mathrm{p}=0.28)$, but a significant effect of day $(\mathrm{F}(4,40)=3.37, \mathrm{p}=0.02)$, however the day effect was due to higher time constants in session 2 and not a general trend. In particular, there was little evidence that adaptation occurred more quickly with repeated exposures.

To explore how the recovery rate of the adaptation depended on the adapting duration, we repeated the settings for 4 observers (not including MW) with a 10 min adapting duration. Despite the substantially shorter exposure, both the strength of the aftereffects and the time constant for the recovery did not significantly differ from the aftereffects with 1 hour exposure (Figure 5). Thus for most observers the adaptation effects appeared to involve only a rapid adjustment, as if the adaptation was providing a roughly "real-time" calibration for the current stimulus history.

Finally, we assessed whether the form of the contrast response change varied with the duration of the adaptation. This analysis was based on comparing the relative changes in perceived contrast at the 3 different test levels, and whether these relative differences shifted during the one hour session. To evaluate this, we estimated the perceived contrast at 6 different intervals during the adaptation period. These began at 150 seconds and were separated by intervals of 570 seconds. Rather than relying on the exponential fits, within 
each interval we calculated the average matches over a period of 300 seconds, averaged across all of the 5 days. These are shown in Figure 6 for 4 representative observers. In this case the plots represent the perceived contrast as a function of the test contrast, while the separate plots show the matches at different points during the adaptation. For a physical match, the curves should lie along the diagonal. Instead, they are shifted below the physical match because perceived contrast was reduced during the adaptation. Again, our interest here was in how it was reduced. A change in contrast gain should roughly reduce the required match contrast by a constant amount and thus reflect a subtractive effect of the adaptation. This prediction is shown by the line labeled "subtractive" in the figure. A change in response gain should instead reduce perceived contrast by a constant ratio (line labeled "multiplicative"). This divisive effect should therefore have the largest absolute change at the highest test contrast. For one observer (SS), the pattern was close to a simple subtractive change, while the remaining observers' matches were intermediate to a subtractive or divisive shift. This pattern is similar to the results reported previously for chromatic contrast adaptation [14]. Moreover, for 3 of the observers the contrast losses do not depend on the adapting interval, and thus did not change in magnitude or form during the hour. For MW, the slow build-up of the adaptation over time is reflected in the ordered shifts in the curves toward lower perceived contrast at the successive sampling intervals. Over the adaptation period this again did not lead to a strong qualitative shift in the form of the adaptation. Importantly however, as noted above, the slow component of MW's adaptation was consistent with a constant relative change at the different contrast levels. If this pattern continued at longer durations than we measured then it would eventually lead to a largely divisive response change.

\section{Discussion}

Our results show rapid changes in perceived contrast with chromatic contrast adaptation, yet give surprisingly little hint of longer term adjustments. In particular, we found no evidence for a carry-over of the adaptation across successive days, nor for a change in the dynamics of the adaptation with repeated exposure. Moreover, we did not observe systematic changes in the form of the aftereffects with increasing adaptation duration. Instead, the aftereffects were notable for both their rapid yet short-lived character. How can these effects be reconciled with the mounting evidence for long-term aftereffects that have been observed with other forms of color adaptation or with luminance contrast adaptation?

First, our results point to a clear distinction between simple contrast changes and long-term color-contingent aftereffects such as the ME. The latter can produce very prolonged aftereffects even with a few minutes of exposure [5]. Again, the bases for these remain shrouded in mystery. However, the present results suggest that the temporal contrast in the stimuli alone is unable to support persistent aftereffects, over the same timescales that generate very robust MEs. One difference in procedure is that we measured the changes in contrast rather than the hue shifts associated with the ME. However, in further informal measurements (not reported), we confirmed that the hue shifts with chromatic contrast adaptation also showed a rapid buildup and recovery similar to the contrast changes. (Specifically, adapting to the LvsM modulation leads to shifts in the appearance of unique yellow consistent with a selective loss in sensitivity to the LvsM chromatic signals, and in the observer tested (KT) this showed a similar time course to the perceived contrast changes.) Our results thus rule out one potential source of the long-term dynamics in the $\mathrm{ME}$, which is also based on a temporal alternation between two colors. While it is not surprising that the ME persistence must therefore also be related to the spatial alternation in the patterns (between two orientations) or to the presence of a contingency (how the color and orientation are paired), the present results provide a baseline for progressively adding stimulus attributes to dissect which specific features are critical for the persistent aftereffects 
to emerge. For example, our measurements were limited to temporal modulations in the color of large uniform fields. It is possible that different dynamics control the adaptation of spatially varying patterns. Further, we tested only equiluminant modulations. One account of the ME is that it specifically reflects adaptation to mechanisms coding color at luminance edges [34].

In relation to other longer-term aftereffects, a limitation of our present work is that the onehour adapting duration is still relatively short. The persistent aftereffects reported for both color and contrast instead have typically required two or more hours to become manifest [7, $24,27,32]$, durations that were not practical for the procedure we used. An exception is in recent studies exploring shifts in unique yellow based on adapting to a random sequence of red lines, which produces robust long-term changes after one hour [8]. However, this stimulus itself has many similarities to the ME, and thus it is not clear whether it is tapping a form of adaptation fundamentally different from the ME. In any case, it remains possible that separate dynamics would have developed for our stimuli had we tested over longer timescales. Thus, we cannot exclude the possibility that the dynamics of luminance and chromatic contrast adaptation are not in fact different. If that is the case, our results reinforce that longer-term dynamics do not begin to become manifest with only an hour of adaptation, which is nevertheless a fairly prolonged stimulus exposure compared to typical adaptation paradigms.

Theoretically, it remains likely that most if not all aspects of visual coding are calibrated over multiple timescales [35-37]. For color contrast this is important because the range of chromatic variation in the environment can vary substantially for different scenes and also changes dramatically within scenes, for example as the seasons change [38]. Short-term adjustments of the kind we observed could rapidly shift contrast coding for different contexts. However, the observed subtractive-like changes in contrast gain alone are not well suited for matching neural responses to changes in the overall gamut of colors [27-29], or for compensating for the sensitivity characteristics of the observer [30, 39]. The fact that the more divisive-like or response-gain changes (that can provide gamut matching) were not found does not suggest they do not occur for color, but may like luminance contrast require more extended sampling by the visual system before there is sufficient evidence to implement them. Our study suggests that for the hour durations over several days that we explored them, the visual system appears to calibrate chromatic contrast based only on the very short-term context, so that the dynamics of the adaptation itself remain highly stable, and thus are driven by only short-term memory of the stimulus history.

\section{Acknowledgments}

Supported by EY-10834.

\section{References}

1. Webster MA. Adaptation and visual coding. Journal of vision. 2011; 11:3, 1-23. [PubMed: 21602298]

2. Wark B, Lundstrom BN, Fairhall A. Sensory adaptation. Curr Opin Neurobiol. 2007; 17:423-429. [PubMed: 17714934]

3. Kohn A. Visual adaptation: physiology, mechanisms, and functional benefits. Journal of neurophysiology. 2007; 97:3155-3164. [PubMed: 17344377]

4. McCollough C. Color Adaptation of Edge-Detectors in the Human Visual System. Science. 1965; 149:1115-1116. [PubMed: 17737844]

5. McCollough-Howard C, Webster MA. McCollough effect. Scholarpedia. 2011; 6(2):8175. 
6. Vul E, Krizay E, MacLeod DI. The McCollough effect reflects permanent and transient adaptation in early visual cortex. Journal of vision. 2008; 8:4, 1-12. [PubMed: 18831617]

7. Neitz J, Carroll J, Yamauchi Y, Neitz M, Williams DR. Color perception is mediated by a plastic neural mechanism that is adjustable in adults. Neuron. 2002; 35:783-792. [PubMed: 12194876]

8. Belmore SC, Shevell SK. Very-long-term chromatic adaptation: test of gain theory and a new method. Visual neuroscience. 2008; 25:411-414. [PubMed: 18598411]

9. Belmore SC, Shevell SK. Very-long-term and short-term chromatic adaptation: are their influences cumulative? Vision research. 2010; 51:362-366. [PubMed: 21130799]

10. Delahunt PB, Webster MA, Ma L, Werner JS. Long-term renormalization of chromatic mechanisms following cataract surgery. Visual neuroscience. 2004; 21:301-307. [PubMed: 15518204]

11. Kitakawa T, Nakadomari S, Kuriki I, Kitahara K. Evaluation of early state of cyanopsia with subjective color settings immediately after cataract removal surgery. Journal of the Optical Society of America A, Optics, image science, and vision. 2009; 26:1375-1381.

12. Webster MA. Human colour perception and its adaptation. Network: Computation in Neural Systems. 1996; 7:587-634.

13. Webster MA, Leonard D. Adaptation and perceptual norms in color vision. Journal of the Optical Society of America A. 2008; 25:2817-2825.

14. Webster MA, Mollon JD. Changes in colour appearance following post-receptoral adaptation. Nature. 1991; 349:235-238. [PubMed: 1987475]

15. Webster MA, Mollon JD. The influence of contrast adaptation on color appearance. Vision research. 1994; 34:1993-2020. [PubMed: 7941399]

16. Krauskopf J, Williams DR, Heeley DW. Cardinal directions of color space. Vision research. 1982; 22:1123-1131. [PubMed: 7147723]

17. Bradley A, Switkes E, De Valois K. Orientation and spatial frequency selectivity of adaptation to color and luminance gratings. Vision research. 1988; 28:841-856. [PubMed: 3227661]

18. Clifford CW, Spehar B, Solomon SG, Martin PR, Zaidi Q. Interactions between color and luminance in the perception of orientation. Journal of vision. 2003; 3:106-115. [PubMed: 12678615]

19. Webster MA, Wilson JA. Interactions between chromatic adaptation and contrast adaptation in color appearance. Vision research. 2000; 40:3801-3816. [PubMed: 11090672]

20. Webster MA, Malkoc G. Color-luminance relationships and the McCollough effect. Percept Psychophys. 2000; 62:659-672. [PubMed: 10883575]

21. Magnussen S, Greenlee MW. Marathon adaptation to spatial contrast: saturation in sight. Vision research. 1985; 25:1409-1411. [PubMed: 4090274]

22. Greenlee MW, Georgeson MA, Magnussen S, Harris JP. The time course of adaptation to spatial contrast. Vision research. 1991; 31:223-236. [PubMed: 2017883]

23. Magnussen S, Johnsen T. Temporal aspects of spatial adaptation. A study of the tilt aftereffect. Vision research. 1986; 26:661-672. [PubMed: 3739240]

24. Zhang P, Bao M, Kwon M, He S, Engel SA. Effects of orientation-specific visual deprivation induced with altered reality. Curr Biol. 2009; 19:1956-1960. [PubMed: 19896377]

25. Bao M, Engel SA. Distinct mechanism for long-term contrast adaptation. Proc Natl Acad Sci U S A. 2012; 109:5898-5903. [PubMed: 22454502]

26. Mesik J, Bao M, Engel SA. Spontaneous recovery of motion and face aftereffects. Vision research. 2013; 89:72-78. [PubMed: 23872167]

27. Kwon M, Legge GE, Fang F, Cheong AM, He S. Adaptive changes in visual cortex following prolonged contrast reduction. Journal of vision. 2009; 9:20, 21-16. [PubMed: 19271930]

28. McDermott, K.; Juricevic, I.; Bebis, G.; Webster, MA. Adapting images to observers. In: Rogowitz, BE.; Pappas, TN., editors. Human Vision and Electronic Imaging, SPIE. 2008. p. V-1-10.

29. Juricevic I, Webster MA. Variations in normal color vision. V. Simulations of adaptation to natural color environments. Visual neuroscience. 2009; 26:133-145. [PubMed: 19203426] 
30. Webster MA, Juricevic I, McDermott KC. Simulations of adaptation and color appearance in observers with varying spectral sensitivity. Ophthalmic Physiol Opt. 2010; 30:602-610. [PubMed: 20883345]

31. Georgeson MA. The effect of spatial adaptation on perceived contrast. Spatial vision. 1985; 1:103112. [PubMed: 3940052]

32. Yehezkel O, Sagi D, Sterkin A, Belkin M, Polat U. Learning to adapt: Dynamics of readaptation to geometrical distortions. Vision research. 2010; 50:1550-1558. [PubMed: 20493205]

33. Webster MA, Miyahara E, Malkoc G, Raker VE. Variations in normal color vision. I. Coneopponent axes. Journal of the Optical Society of America A, Optics, image science, and vision. 2000; 17:1535-1544.

34. Broerse J, Vladusich T, O'Shea RP. Colour at edges and colour spreading in McCollough effects. Vision research. 1999; 39:1305-1320. [PubMed: 10343844]

35. Kording KP, Tenenbaum JB, Shadmehr R. The dynamics of memory as a consequence of optimal adaptation to a changing body. Nature neuroscience. 2007; 10:779-786.

36. Shadmehr R, Smith MA, Krakauer JW. Error correction, sensory prediction, and adaptation in motor control. Annu Rev Neurosci. 2010; 33:89-108. [PubMed: 20367317]

37. Wark B, Fairhall A, Rieke F. Timescales of inference in visual adaptation. Neuron. 2009; 61:750761. [PubMed: 19285471]

38. Webster MA, Mizokami Y, Webster SM. Seasonal variations in the color statistics of natural images. Network. 2007; 18:213-233. [PubMed: 17926193]

39. von der Twer T, MacLeod DI. Optimal nonlinear codes for the perception of natural colours. Network. 2001; 12:395-407. [PubMed: 11563536] 

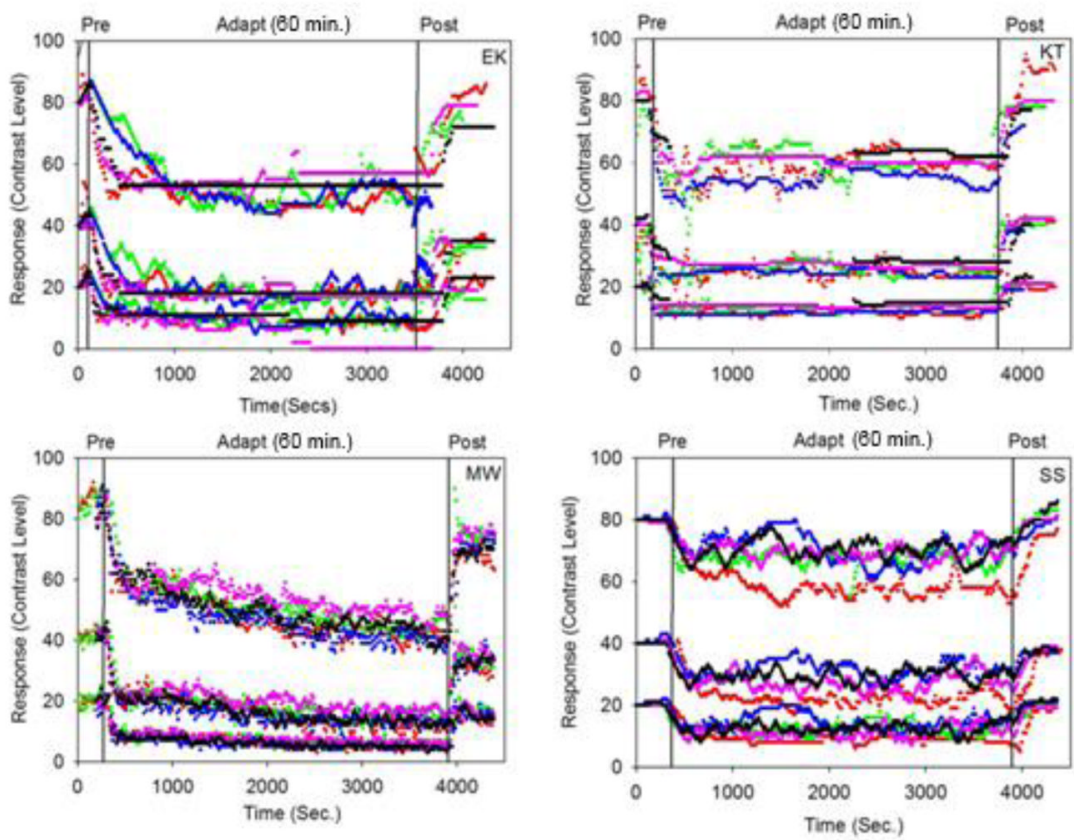

Figure 1.

Contrast matches during the daily adapting sessions. Panels show the settings for 4 individual observers, and plot the matches to each of the three test contrasts $(20,40$, or 80$)$ either before (pre), during (adapt) or after (post) exposure to the adapting stimulus. Different color traces correspond to repeated settings over 5 days: day 1 (red); day 2 (green); day 3 (blue); day 4 (pink); day 5 (black). 

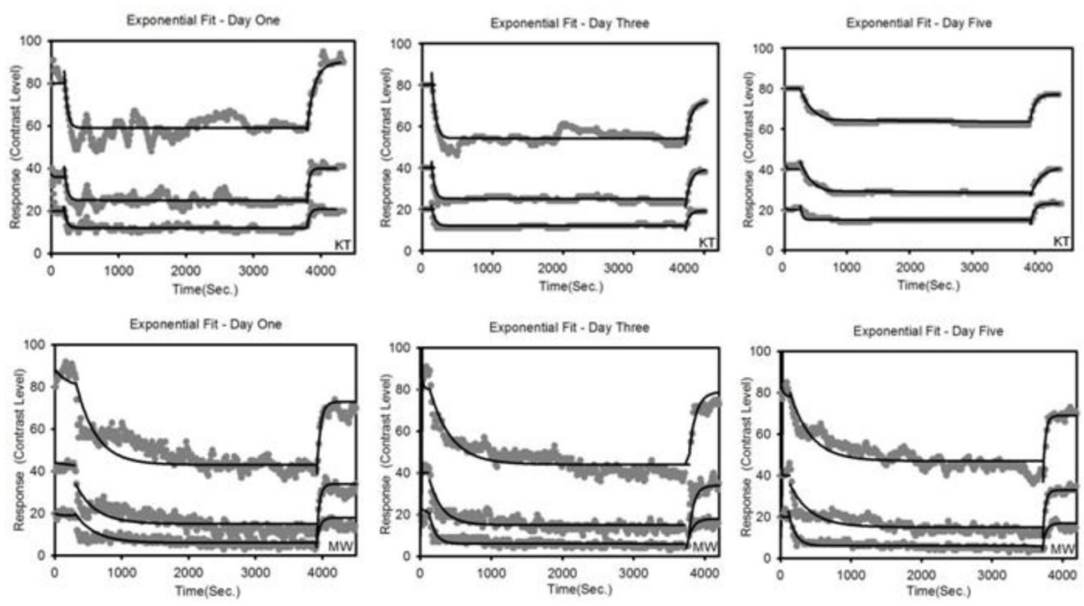

Figure 2.

Examples of exponential fits (black lines) to the contrast matches (gray traces) for KT (top) or MW (bottom). Separate exponentials were fit to the pre, post, and adapt phases. These characterized the changes in perceived contrast for most observers (like KT), but do not approximate the dynamics during the adaptation for MW. 

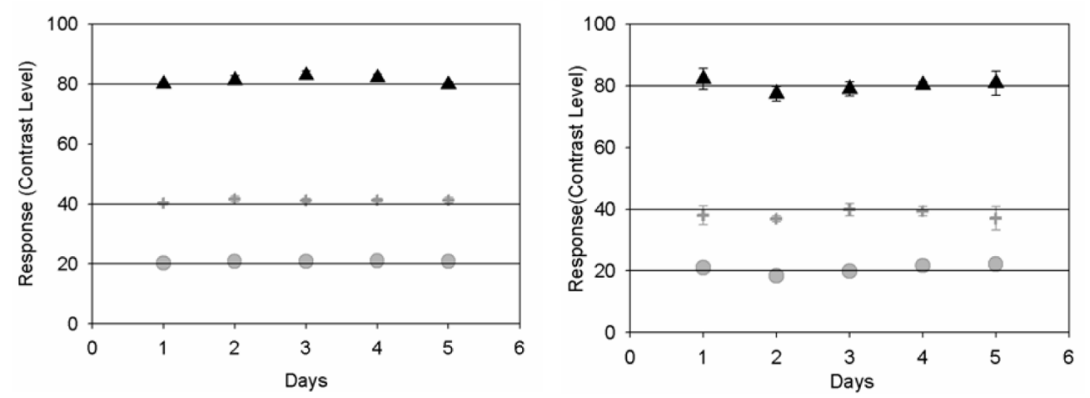

Figure 3.

Mean contrast matches (across observers) \pm 1 SE either at the end of the pre-adapt phase (left) or at the end of the post-adapt phase (right). The three sets of symbols correspond to the test contrasts of 20,40, or 80 measured over the 5 sessions. Matches returned to a physical match by the end of the post-adapt phase and showed no carry-over of aftereffects at the beginning of the next daily session. 

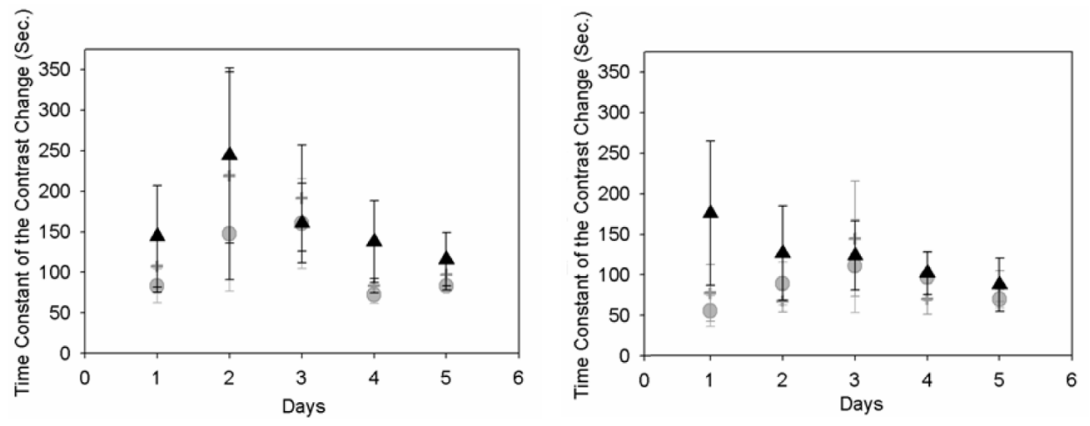

Figure 4.

Time constants for the perceived contrast changes for the 5 daily sessions, either during the adaptation period (left) or during the post-adapt recovery (right). Symbols plot the mean time constants across observers $\pm 1 \mathrm{SE}$, for test contrasts of 20 (circle), 40 (plus), or 80 (triangle). 

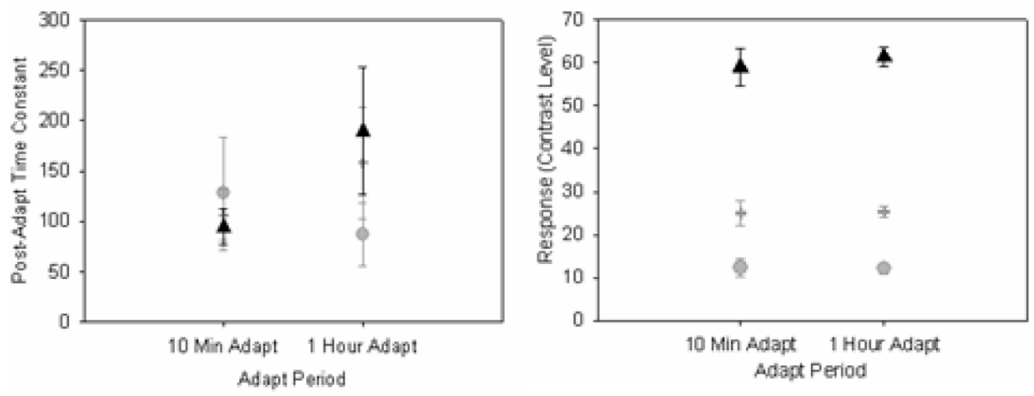

Figure 5.

Mean time constants (across observers and sessions) \pm 1 SE for the post adapt phase (left), and mean minimum contrast matches (across observers and sessions) \pm 1 SE during the adapt phase (right), each for test contrasts of 20 (circle), 40 (plus), or 80 (triangle). 

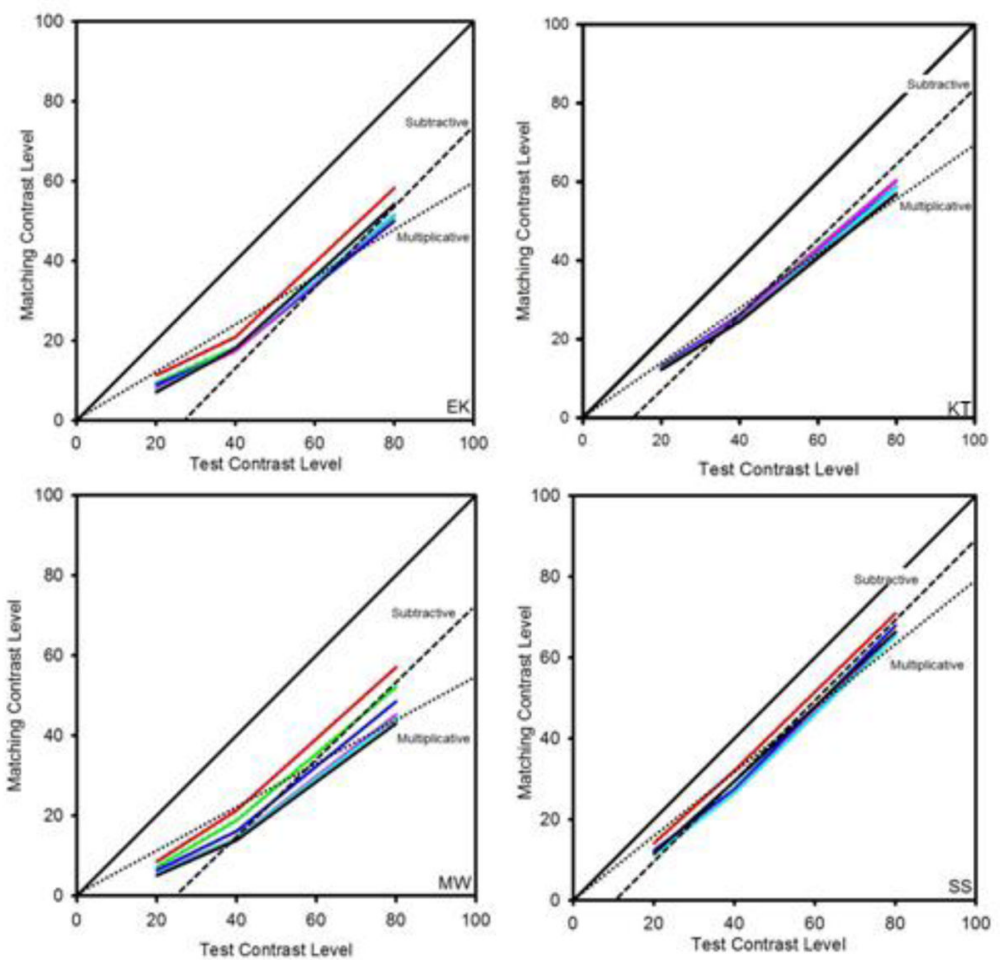

Figure 6.

Matching contrast during adaptation as a function of the test contrast. Each panel plots the settings for a different observer. Lines show the perceived contrast at 6 successive times sampled at $\sim 10$ minute intervals. Time 1 (red); time 2 (green); time 3 (blue); time 4 (purple); time 5 (cyan); time 6 (black). 